SLAC-PUB-8143

May 1999

\title{
ELECTROWEAK RESULTS FROM THE SLD EXPERIMENT**
}

\author{
M. Woods \\ Stanford Linear Accelerator Center, \\ Stanford University, Stanford, CA 94309 \\ Representing
}

The SLD Collaboration

\begin{abstract}
We present an overview of the electroweak physics program of the SLD experiment at the Stanford Linear Accelerator Center (SLAC). A data sample of $550 \mathrm{~K} Z^{0}$ decays has been collected. This experiment utilizes a highly polarized electron beam, a small interaction volume, and a very precise pixel vertex detector. It is the first experiment at a linear electron collider. We present a preliminary result for the weak mixing angle, $\sin ^{2}\left(\theta_{W}^{\text {eff }}\right)=0.23110 \pm 0.00029$. We also present a preliminary result for the parity violating parameter, $A_{b}=0.898 \pm 0.029$. These measurements are used to test for physics beyond the Standard Model.
\end{abstract}

Presented at the Lake Louise Winter Institute: Electroweak Physics

Lake Louise, Alberta, Canada

February 14-20, 1999

*Work supported by Department of Energy contract DE-AC03-76SF00515 


\section{Electroweak Physics program of the SLD Experiment}

The SLD Experiment began its physics program at the SLAC Linear Collider (SLC) in 1992, and has accumulated a total data sample of approximately 550K hadronic $Z^{0}$ decays between 1992 and 1998. This data sample is a factor 30 smaller than the $Z^{0}$ sample available from the combined data of the 4 LEP experiments, ALEPH, DELPHI, L3 and OPAL. Yet the SLD physics results in many areas are competitive with the combined LEP result, and for some measurements SLD has the world's most precise results.

There are 3 features that distinguish the SLD experiment at the SLC: a small, stable interaction volume; a precision vertex detector; and a highly polarized electron beam. SLD is the first experiment at an electron linear collider. The collision volume is small and stable, measuring 1.5 microns by 0.7 microns in the transverse dimensions by 700 microns longitudinally.

These key features for the SLD experiment result in the world's best measurement of the weak mixing angle, a precise direct measurement of parity violation at the $Z b \bar{b}$ vertex, $A_{b}$, and a good measurement of the $Z b \bar{b}$ coupling strength, $R_{b}$. The weak mixing angle measurement provides an excellent means to search for new physics that may enter through oblique (or loop) corrections, while the $A_{b}$ and $R_{b}$ measurements are excellent means to search for new physics that may enter through a correction at the $Z b \bar{b}$ vertex.

In its near (analysis) future, SLD is also exploiting its capabilities to search for $B_{s}$ mixing. The analysis for this is evolving to take full advantage of the precise vertexing information, and by the time of the summer 1999 conferences SLD should have a measurement of $B_{\boldsymbol{g}}$ mixing comparable in sensitivity with the combined LEP result. SLD estimates it should have a reach for $\Delta m_{g}$ of $12-15 p s^{-1}$, in the region where it is predicted in the SM. 


\section{$2 Z^{0}$ Coupling Parameters}

At the $Z f \bar{f}$ vertex, the SM gives the vector and axial vector couplings to be $v_{f}=I_{f}^{3}-2 Q_{f} \sin ^{2}\left(\theta_{W}^{e f f}\right)$, and $a_{f}=I_{f}^{3}$, where $I_{f}$ is the fermion isospin and $Q_{f}$ is the fermion charge. Radiative corrections are significant and are treated as follows. First, vacuum polarization and vertex corrections are included in the coupling constants, and an effective weak mixing angle is defined to be $\sin ^{2}\left(\theta_{W}^{\text {eff }}\right) \equiv \frac{1}{4}\left(1-v_{e} / a_{e}\right)$. Second, experimental measurements need to be corrected for initial state radiation and for $Z-\gamma$ interference to extract the $Z$-pole contribution.

One can define a parity-violating fermion asymmetry parameter, $A_{f}=$ $\frac{2 v_{f} a_{f}}{v_{f}^{2}+a_{f}^{2}}$. The cross-section for $e^{+} e^{-} \rightarrow Z^{0} \rightarrow f \bar{f}$ can be expressed by

$$
\frac{d \sigma^{f}}{d \Omega} \propto\left[v_{f}^{2}+a_{f}^{2}\right]\left\{\begin{array}{c}
\left(1+\cos ^{2} \theta\right)\left(1+P A_{e}\right)+ \\
2 \cos \theta A_{f}\left(P+A_{e}\right)
\end{array}\right\}
$$

where $\theta$ is the angle of the outgoing fermion with respect to the incident electron, and $P$ is the polarization of the electron beam (the positron beam is assumed to be unpolarized). We can then define forward, backward, and left, right cross-sections as follows: $\sigma_{F}=\int_{0}^{1} \frac{d \sigma}{d \Omega} d(\cos \theta) ; \sigma_{B}=\int_{-1}^{0} \frac{d \sigma}{d \Omega} d(\cos \theta)$; $\sigma_{L}=\int_{-1}^{1} \frac{d \sigma_{L}}{d \Omega} d(\cos \theta) ; \sigma_{R}=\int_{-1}^{1} \frac{d \sigma_{R}}{d \Omega} d(\cos \theta)$. Here, $\sigma_{L}\left(\sigma_{R}\right)$ is the cross-section for left (right) polarized electrons colliding with unpolarized positrons.

At the SLC, the availability of a highly polarized electron beam allows for direct determinations of the $A_{f}$ parameters via measurements of the left-right forward-backward asymmetry, $A_{L R}^{F B}$, defined by

$$
A_{L R}^{F B}=\frac{\left(\sigma_{F}^{L}-\sigma_{F}^{R}\right)-\left(\sigma_{B}^{L}-\sigma_{B}^{R}\right)}{\sigma_{F}^{L}+\sigma_{F}^{R}+\sigma_{B}^{L}+\sigma_{B}^{R}}=\frac{3}{4} P_{\mathrm{e}} A_{f}
$$

Additionally, a very precise determination of $A_{e}$ is achieved from the measurement of the left-right asymmetry, $A_{L R}$, which is defined as

$$
A_{L R}=\frac{1}{P_{e}} \cdot \frac{\sigma_{L}-\sigma_{R}}{\sigma_{L}+\sigma_{R}}=A_{e}
$$

All $Z$ decay modes can be used, and this allows for a simple analysis with good statistical power for a precise determination of $\sin ^{2}\left(\theta_{W}^{e f f}\right)$. 


\section{The SLAC Linear Collider}

LEP200 is the last of the large electron storage rings, and a new technology is needed to push to higher center-of-mass energies. Electron linear collider technology provides a means to achieve this, and the SLC is a successful prototype for this. It has reached a peak luminosity of $3 \cdot 10^{30} \mathrm{~cm}^{-2} \mathrm{~s}^{-1}$, which is within a factor two of the design luminosity ${ }^{1}$. The spotsizes at the Interaction Point (IP) are actually significantly smaller than design, and Fig-

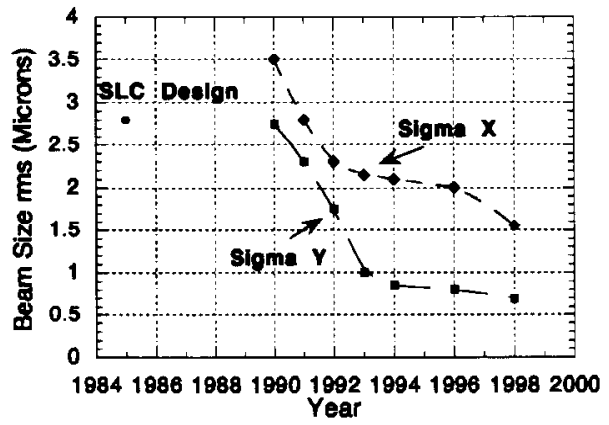

Figure 1: Spotsizes at the SLC. ure 1 indicates how the spotsizes have improved with time. With the small spotsizes, there is an additional luminosity enhancement from the "pinch effect" the two beams have on each other. At the higher luminosities achieved in the last SLC run, the pinch effect enhanced the luminosity by a factor of two. The luminosity is limited due to the maximum charge achievable in single bunches because of instabilities in the Damping Rings.

\section{SLD's Vertex Detector}

SLD's vertex detector ${ }^{2}$ consists of 3 layers, with the inner layer located at a radius of $2.7 \mathrm{~cm}$. Angular coverage extends out to $\cos (\theta)=0.90$. It has 307 million pixels, with a single hit resolution of 4.5 microns. There are $0.4 \%$ radiation lengths per layer. The capability of SLD's vertex detector is illustrated in Figure 2, which is a histogram of the reconstructed jet mass. With a mass cut of $2.0 \mathrm{GeV} / \mathrm{c}^{2}$, SLD can identify b jets with $50 \%$ efficiency and $98 \%$ purity.

\section{SLD's Compton Polarimeter}

This polarimeter, ${ }^{3}$ shown in Figure 3 , detects both Compton-scattered electrons and Compton-scattered gammas from the collision

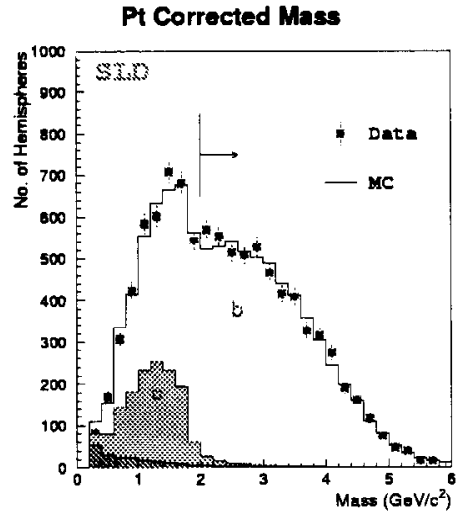

Figure 2: Vertex mass. 
of the longitudinally polarized $45.6 \mathrm{GeV}$ electron beam ${ }^{4}$ with a circularly polarized photon beam. The photon beam is produced from a pulsed Nd:YAG laser with a wavelength of $532 \mathrm{~nm}$. After the Compton Interaction Point (CIP), the electrons and backscattered gammas pass through a dipole spectrometer. A nine-channel threshold Cherenkov detector (CKV) measures electrons in the range 17 to $30 \mathrm{GeV} .^{5}$ Two detectors, a single-channel Polarized Gamma Counter (PGC) ${ }^{6}$ and a multi-channel Quartz Fiber Calorimeter (QFC), ${ }^{7}$ measure the counting rates of Compton-scattered gammas.

Due to beamstrahlung backgrounds produced during luminosity running, only the CKV detector can make polarization measurements during beam collisions. Hence it is the primary detector and the most carefully analyzed. Its systematic error is estimated to be $0.7 \%$. Dedicated electron-only runs are used to compare electron polarization measurements between the CKV, PGC and QFC detectors. The $P G C$ and QFC results are consis-

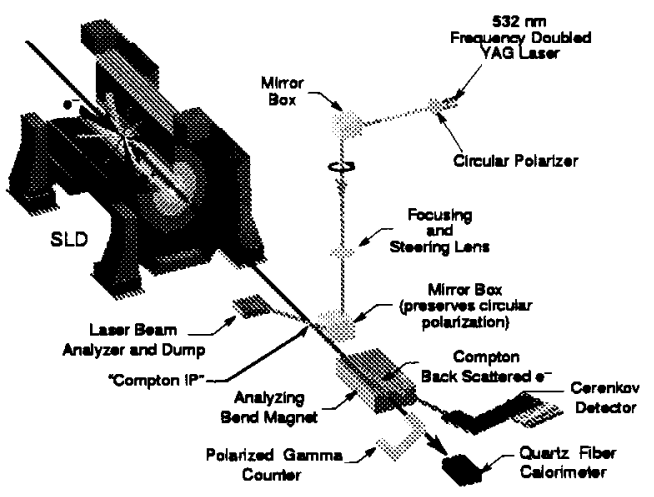

Figure 3: SLD and the Compton Polarimeter. tent with the CKV result at the level of $0.5 \%$. Typical beam polarizations for the SLD experiment have been in the range $73-78 \%$.

\section{Measurements of $\sin ^{2}\left(\theta_{W}^{e f f}\right)$, and testing oblique corrections}

For the $A_{L R}$ analysis, all $Z$ decay modes can be used, though in practice the leptonic modes are excluded. They are analyzed separately in the measurements of $A_{L R}^{F B}$ described below. The $A_{L R}$ event selection requires at least 4 charged tracks originating from the IP and greater than $22 \mathrm{GeV}$ energy deposition in the calorimeter. Energy flow in the event is required to be balanced by requiring the normalized energy vector sum be less than 0.6 . These criteria have an efficiency of $\mathbf{9 2 \%}$ for hadronic events, with a residual background of $0.1 \%$.

SLD's 1998 running yielded $225 \mathrm{~K}$ hadronic $\mathrm{Z}$ decays, with $N_{L}=124,404$ produced from the left-polarized beam and $N_{R}=100,558$ produced from the right-polarized beam. For the measured beam polarization of $73.1 \%$, this yielded $A_{L R}^{\text {meas }}=0.1450 \pm 0.0030($ stat $)$. Correcting for initial state radiation and $Z-\gamma$ interference effects, gives $A_{L R}^{0}=0.1487 \pm 0.0031$ (stat) \pm 0.0017 (syst). 
The systematic error includes a contribution of 0.0015 from uncertainties in the polarization scale and 0.0007 from uncertainties in the energy scale. This result determines the weak mixing angle to be $\sin ^{2}\left(\theta_{W}^{e f f}\right)=0.23130 \pm 0.00039 \pm$ 0.00022 . Combining all of SLD's $A_{L R}$ results from 1992-98, gives $\sin ^{2}\left(\theta_{W}^{e f f}\right)=$ $0.23101 \pm 0.00031$.

For the $A_{L R}^{F B}$ analysis, leptonic $Z$ decay events are selected as follows. The number of charged tracks must be between 2 and 8 . One hemisphere must have a charge of -1 and the other hemisphere a charge of +1 . The polar angle is required to have $\cos (\theta)<0.8$. For ee final state events, the only additional requirement is a deposition of greater than $45 \mathrm{GeV}$ in the calorimeter. The $\mu \mu$ final state events must reconstruct with a large invariant mass and have less than $10 \mathrm{GeV}$ per track deposited in the calorimeter. The $\tau \tau$ final state events must reconstruct with an invariant mass less than $70 \mathrm{GeV}$, and deposit less than $27.5 \mathrm{GeV}$ per track in the calorimeter. One stiff track is required $(>3 \mathrm{GeV})$, the acollinearity angle must be greater than $160^{\circ}$ and the invariant mass in each hemisphere must be less than $1.8 \mathrm{GeV}$. Event selection efficiencies are $87.3 \%$

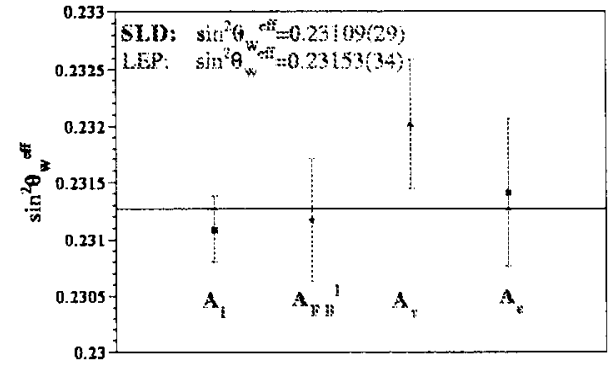

Figure 4: Weak Mixing angle measurements.

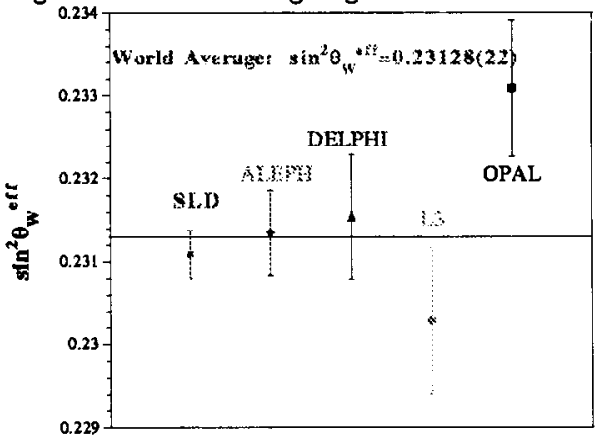

Figure 5: Weak Mixing angle measurements. for $e e, 85.5 \%$ for $\mu \mu$, and $78.1 \%$ for $\tau \tau$. Backgrounds are estimated to be $1.2 \%$ for $e e$ (predominantly $\tau \tau$ ), $0.2 \%$ for $\mu \mu$ (predominantly $\tau \tau$ ), and $5.2 \%$ for $\tau \tau$ (predominantly $\mu \mu$ and $2 \gamma$ ).

We use Equation 1 in a maximum likelihood analysis (which also allows for photon exchange and for $Z-\gamma$ interference) to determine $A_{e}, A_{\mu}$ and $A_{\tau}$. The results are $A_{e}=0.1504 \pm 0.0072, A_{\mu}=0.120 \pm 0.019$, and $A_{\tau}=0.142 \pm$ 0.019 . These results are consistent with universality and can be combined, giving $A_{e, \mu, \tau}=0.1459 \pm 0.0063$. This determines the weak mixing angle to be $\sin ^{2}\left(\theta_{W}^{e f f}\right)=0.2317 \pm 0.0008$.

Combining the $A_{L R}$ measurements that use hadronic final states and the $A_{L R}^{F B}$ measurements that use leptonic final states, we determine the weak mix- 
ing angle to be $\sin ^{2}\left(\theta_{W}^{\text {eff }}\right)=0.23110 \pm 0.00029$. This is a preliminary result.

A comparison of SLD's result with leptonic asymmetry measurements at LEP $^{8}$ is given in Figure 4. These results are compared by technique, where $A_{l}$ is SLD's combined result from $A_{L R}$ and $A_{L R}^{F B}$ (leptons) described above; $A_{F B}^{l}$ is the LEP result using the forward-backward asymmetry with leptonic final states; $A_{\tau}$ and $A_{e}$ are the LEP results from analyzing the $\tau$ polarization for the $\tau \tau$ final state. We do not include in this comparison the LEP results using hadronic final states. These results are discussed below, when we examine SLD's $A_{b}$ measurement and tests of $b$ vertex corrections. The SLD and LEP data in Figure 4 are replotted in Figure 5 by experiment rather than by technique. The data are consistent and can be combined to give a world average $\sin ^{2}\left(\theta_{W}^{e f f}\right)=0.23128 \pm 0.00022$.

A convenient framework for analyzing the consistency of the $\sin ^{2}\left(\theta_{W}^{e f f}\right)$ measurement with the SM and with other electroweak measurements is given by the PeskinTakeuchi parametrization ${ }^{9}$ for probing extensions to the SM. This parametrization assumes that vacuum polarization effects dominate and expresses new physics in terms of the parameters $S$ and $T$, which are defined in terms of the self-energies of the gauge bosons. In $S$ - $T$ space, a measurement of an electroweak ob-

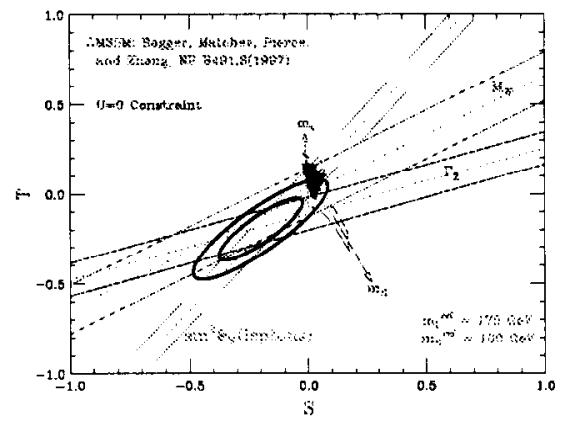

Figure 6: Testing oblique corrections. servable corresponds to a band with a given slope. Figure 6 shows the $S-T$ plot for measurements of the weak mixing angle $\left(\sin ^{2}\left(\theta_{W}^{e f f}\right)\right)$, the $Z$ width $\left(\Gamma_{Z}\right),{ }^{8}$ and the $W$ mass $\left(M_{W}\right),{ }^{8}$. The experimental bands shown correspond to one sigma contours. The elliptical contours are the error ellipses $(68 \%$ confidence and $\mathbf{9 5 \%}$ confidence) for a combined fit to the data. The SM allowed region is the small parallelogram, with arrows indicating the dependence on $m_{t}$ and $m_{H}$. The Higgs mass is allowed to vary from $100 \mathrm{GeV}$ to $1000 \mathrm{GeV}$ and $m_{t}$ from $165 \mathrm{GeV}$ to $185 \mathrm{GeV}$. The measurements are in reasonable agreement with the SM and favour a light Higgs mass. A comparison is also given to a prediction for the parameter space of the Minimal Supersymmetric Model ${ }^{10}$ (region of dots in figure). The combined SLD and LEP measurement for the weak mixing angle gives the narrowest band in $S-T$ space, and provides the best test of the SM for oblique corrections. Improved measurements of $M_{W}$ from LEP and FNAL are eagerly awaited to further constrain and test the SM 
in this regard.

\section{Measurements of $A_{b}$, and testing vertex corrections}

The measurement technique for determining $A_{b}$ is similar to that for determining $A_{e}, A_{\mu}$ and $A_{\tau}$. For this analysis, the capabilities of SLD's vertex detector is critical and good use is also made of SLD's particle identification system to identify kaons with the Cherenkov Ring Imaging Detector (CRID). Three different analyses are employed with different techniques for determining the $b$-quark charge. The Jet Charge analysis uses a momentumweighted jet charge to identify the $b$ quark charge, and it requires a sec-

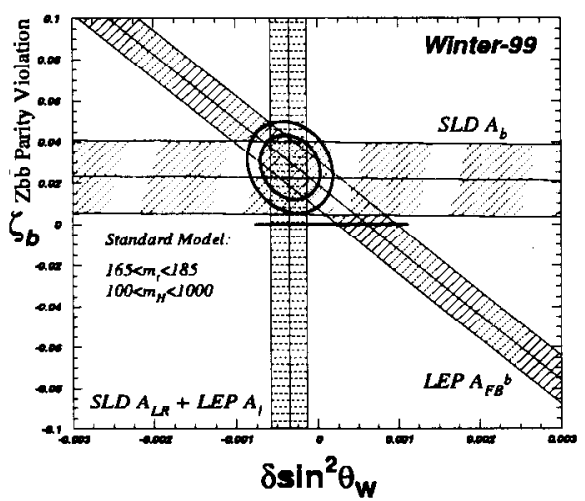

Figure 7: Testing vertex corrections. ondary vertex mass greater than $2.0 \mathrm{GeV}$. The Kaon Tag analysis uses the kaon sign in the cascade decay $(b \rightarrow c \rightarrow s)$ to identify the $b$-quark charge, and it requires a secondary vertex mass greater than $1.8 \mathrm{GeV}$. The Lepton Tag analysis uses the lepton charge in semileptonic decays to identify the $b$-quark charge; it has no secondary vertex mass requirement.

The three analyses yield the following results: $A_{b}$ (Jet Charge) $=0.882 \pm$ $0.020 \pm 0.029 ; A_{b}(\mathrm{KaOn} \mathrm{Tag})=0.855 \pm 0.088 \pm 0.102 ;$ and $A_{b}$ (Lepton Tag) $=$ $0.924 \pm 0.032 \pm 0.026$. These results can be combined, giving $A_{b}=0.898 \pm 0.029$. This is a preliminary result.

Similar to the $S-T$ analysis for testing oblique corrections, one can utilize an extended parameter space for testing vertex corrections. This is done in Figure 7, where we plot the deviation in $A_{b}$ from the SM prediction versus the deviation in $\sin ^{2}\left(\theta_{W}^{\text {eff }}\right)$ from the SM prediction. The three bands plotted are SLD's $A_{b}$ measurement, the combined leptonic $\sin ^{2}\left(\theta_{W}^{\text {eff }}\right)$ measurement from SLD and LEP, and LEP's forward-backward $b$ asymmetry. The elliptical contours are the error ellipses (68\% confidence and $95 \%$ confidence) for a combined fit to the data. The horizontal line is the SM prediction. We note that the data are in excellent agreement, but differ from the SM prediction by $2.6 \sigma$. Unfortunately, there will be no new data to indicate whether this deviation results from a statistical fluctuation, a problem in the $b$ physics analysis, or 
new physics. We also note the discrepancy of where the SLD-LEP $\sin ^{2}\left(\theta_{W}^{e f f}\right)$ and the LEP $A_{F B}^{b}$ measurement bands intersect the SM line. This reflects their $2.2 \sigma$ discrepancy in determining $\sin ^{2}\left(\theta_{W}^{e f f}\right)$ within the SM framework.

\section{Conclusions}

The SLD experiment has been the first experiment at an electron linear collider. The viability of a linear collider has been demonstrated and this technology is now being proposed for future $e^{+} e^{-}$colliders with center-of-mass energies up to $1 \mathrm{TeV}$. The SLD has made many important contributions to precision electroweak physics. SLD has made the best measurement of the weak mixing angle, $\sin ^{2}\left(\theta_{W}^{\text {eff }}\right)=0.23110 \pm 0.00029$ (preliminary). This provides a stringent test of oblique corrections; our measurement is consistent with SM predictions and favours a light Higgs mass. SLD makes the only direct measurement of $A_{b}$, which we determine to be $A_{b}=0.898 \pm 0.029$ (preliminary). This measurement, together with measurements by SLD and LEP of $\sin ^{2}\left(\theta_{W}^{e f f}\right)$ and LEP's measurement of $A_{F B}^{b}$, can be used to test $b$ vertex corrections. The data are consistent, but indicate a $2.6 \sigma$ discrepancy with the SM prediction.

\section{References}

1. P. Raimondi et al., SLAC-PUB-7955 (1998).

2. K. Abe et al., Nucl. Instrum. Methods A 400, 287 (1997).

3. M. Woods in SPIN96 Proceedings, ed. C.W. de Jager et al. (World Scientific, Singapore, 1997), p.843.

4. M. Woods in SPIN96 Proceedings, ed. C.W. de Jager et al. (World Scientific, Singapore, 1997), p.623.

5. M. Fero et al., SLD-Physics-Note-50 (1996).

6. R.C. Field et al., IEEE Trans. Nucl. Sci. 45, 670 (1998).

7. S.C. Berridge et al., in Calorimetry in High Energy Physics Proceedings, ed. E. Cheu et al. (World Scientific, Singapore, 1998), p. 170.

8. D. Abbaneo et al., CERN-EP-99-015 (1999).

9. M.E. Peskin and T. Takeuchi, Phys. Rev. D 46, 381 (1992)

10. J. Bagger et al., Nucl. Phys. B 491, 3 (1997).

11. T. Takeuchi et al., published in DPF 94 Proceedings, ed. S. Seidel (World Scientific, Singapore, 1995), p. 1231. 